\title{
Chronic exertional compartment syndrome
}

\author{
Mark Hutchinson
}

\section{Correspondence to \\ Mark Hutchinson, Department of Orthopaedics, University of Illinois at Chicago, \\ Chicago, Illinois, USA; \\ mhutch@uic.edu}

Accepted 21 April 2011

Published Online First

25 May 2011

\section{INTRODUCTION}

The diagnosis of chronic exertional compartment syndrome (CECS) is commonly delayed due to the poor use of terminology by athletes, and many clinicians, that virtually any pain in the leg is 'shin splints'. We will be able to provide our patients with a better service if we can target a more specific diagnosis with earlier and focused treatment. Therefore, when the history and presentation are most consistent with a stress fracture, radiographs and possibly an MRI or a bone scan should be obtained. If the pain is posterior and related to blood flow, venous or arterial Doppler studies or magnetic resonance angiography should be obtained to confirm the diagnosis of a blood clot or popliteal artery syndrome. Finally, when the history includes increasing pain with exertion with associated resolution with rest, the working diagnosis of CECS should be confirmed with intracompartmental pressure testing. Although alternative techniques using infrared sensors have been proposed, ${ }^{1}$ most experts agree that intracompartmental pressure testing is the gold standard.

Key questions, however, remain regarding the specific protocol a clinician should undergo when performing intracompartmental pressure testing. Should both legs be tested? Should the isolated, most symptomatic compartment or all four compartments be routinely tested? Are resting, immediate postexertion and delayed postexertion tests required for adequate testing?

\section{HISTORICAL PROTOCOLS}

Classic use of intracompartmental pressure measurements was established for the diagnosis of acute and not CECSs. In general, the clinical presentation for an acute compartment syndrome is more obvious with exquisite, unrelenting pain associated with a palpably tense compartment. In these cases, it is reasonable to test only the suspicious compartment. Indeed, it is within the standard of care to proceed with a fascial release in the acute setting even without confirming elevated pressures with an intracompartmental pressure measurement. For CECS, the standard of care is to confirm the diagnosis with intracompartmental pressure measurements. A study done in the UK reported that $83 \%$ of clinicians use intracompartmental pressure testing to confirm the diagnosis. ${ }^{2}$

The classic reference for exertional compartment testing can be credited to Pedowitz and colleagues. ${ }^{3}$ They provided the definitions of a positive test for exertional compartment syndromes, which have been the reference for over two decades: resting pressures $>15 \mathrm{~mm} \mathrm{Hg}$, postexertion pressures $>30 \mathrm{~mm} \mathrm{Hg}$ and delayed pressure measurements $>20 \mathrm{~mm} \mathrm{Hg}$ (criteria with
95\% CI). Most clinicians also base their diagnosis on an increase of $>10 \mathrm{~mm} \mathrm{Hg}$ compared with the resting pressure.

\section{DO WE NEED ALL THREE TESTS IN A GIVEN COMPARTMENT (PRE, POST AND DELAYED)?}

It is generally accepted that the immediate postexertion intracompartmental pressure measurement is the best measure to confirm the diagnosis of exertional compartment syndrome. The need for pre and delayed measurements has been debated. Gracia-Mata and colleagues ${ }^{4}$ in their study on adolescents in Spain routinely performed pre, post, 5-min delayed and 15-min delayed testing. In their 2003 review article, Fraipont and Adamson ${ }^{5}$ discussed the various positive criteria at rest, postexertion and delayed but did not make a definitive recommendation that all or only exertional measurements were necessary.

My routine is to obtain resting pre-exertional measurements and immediate postexertion measurements. I believe that observing an increase from the baseline is important in confirming and witnessing the effect of exertion on the process. On many occasions, I have seen resting pressures of $15-19 \mathrm{~mm} \mathrm{Hg}$ never changing to become postexertion positive; on rare occasions, I have seen a pre-exertion measure as high as 25-30 $\mathrm{mm} \mathrm{Hg}$ actually go down with exercises. Each of these would seem to argue against the use of pre-exertional measures; however, I have also seen a number of cases in which the pre-exertion measure was 5-9 $\mathrm{mm} \mathrm{Hg}$ and the postexertion measure jumped up to $25-29 \mathrm{~mm} \mathrm{Hg}$. In these cases, the absolute final pressure would not have qualified for surgical release but the change in pressure $(\Delta \mathrm{P})$ clearly indicates an exertional effect of the pressures and $90 \%$ of these patients do well with surgical release. Historically, I also calculated delayed measures on all patients, but ultimately found that these measures did not affect my clinical plans. My routine is to get pre- and postexertion measures on all patients.

\section{WHY TEST ALL FOUR COMPARTMENTS?}

Exertional compartment syndromes tend to be more subtle with less obvious physical findings than acute compartment syndromes. Indeed, it is often impossible by clinical presentation alone to clarify if a single or adjacent compartment is involved. I have found from having performed hundreds of tests over 17 years of practice that patients with anterolateral leg pain, more commonly than not, will turn out to have elevated pressures in both the anterior and lateral compartments despite their primary symptoms being in only one compartment. Although minimal 
symptomatic involvement of the deep posterior is less common, missing the diagnosis will lead to a postoperative failure. Isolated release of a single compartment leads to the risk of failure or recurrence in many of these patients as the untreated compartment becomes more symptomatic. The measurement of success should be symptom-free return to sport after surgical fascial release with no need to return for a second surgery. No patient ever wants to have to go back to surgery again due to a missed/delayed diagnosis of a compartment that went untested and later became symptomatic. When I test all four compartments in every patient, the patient is keenly aware that he is accepting an extra needle poke so as to reduce his risk of ever having to come back for a second surgery.

\section{DO WE NEED TO TEST BOTH LEGS?}

Clearly, an astute clinician must consider all diagnoses in each leg before instituting treatment. Overlapping pathologies such as stress fractures and medial tibial stress syndrome with CECS are common. Indeed, in many cases, if the associated pathology is treated, symptoms of CECS will defervesce and the surgical release of the pressure positive, exertional compartment syndrome can become unnecessary. Nonetheless, if associated pathologies are not present, it must be acknowledged that CECS may be bilateral in $75-90 \%$ of the patients. Therefore, if you do not test both legs, you are missing the diagnosis in at least three quarters of your patients!

It has been argued that if one symptomatic compartment is positive then you can assume the contralateral symptomatic leg is also positive. The question is which compartment? Is it always right anterior-left anterior; right lateral-left lateral? No one knows. Indeed, if we follow this logic and we know that $75-90 \%$ are bilateral, it follows directly that we would be doing unnecessary surgery on $10-25 \%$ of patients! As for me, I leave nothing to chance or guess work. If I am going to perform a fascial release with its inherent risks of complication, I want to know absolutely that I have the correct diagnosis for each compartment involved.

\section{SO WHAT IS THE DANGER OF INTRACOMPARTMENTAL PRESSURE TESTING?}

It must be admitted that anytime one breaks the skin, infection is a risk. This complication is extraordinarily rare when the skin is prepped for any of a myriad of needle pokes ranging from blood draws, therapeutic injections, joint aspiration and dry needle therapeutic technique. In over 1000 compartments tested in my career, I have never seen an infection or a neurologic injury as a complication of testing. Local bleeding is not uncommon at the time of the study, yet it usually responds to simple pressure and rarely leads to any visible sign of ecchymosis much less haematoma.

If there is a hazard or downside of multiple needle pokes at multiple sites, it is that the patient experiences more pain with the testing. I routinely educate my patients that it is rare to have patients request a repeat of the tests at a later point in time. Patients invariably accept the increased pain of multiple pokes when they understand that it improves the diagnostic accuracy and likely increases their chance of success and reduces the risk of a second surgery. Nobody likes needle pokes but there are some pearls that the clinician can use to make the testing more tolerable to the patient.

1. Use a local anaesthetic with a small 27-gauge needle to numb up the entry sites for the larger bore needle. The anaesthetic should be capable of penetrating the subcutaneous tissue but not the compartment.

2. Let the anesthetic take effect for a few minutes before proceeding with the larger bore needle required for testing.

3. Have the patient try to relax their muscles when testing. It hurts more when they tense up.

4. I routinely use only one skin puncture to test deep and superficial posterior compartments and another puncture to test the anterior and lateral compartments. I simply redirect my needle outside the fascia but in the subcutaneous tissue.

5. I re-use the same skin puncture sites when the patient returns for postexertional measurements.

\section{CONCLUSION}

The goal of a good clinician should be to make an accurate diagnosis that will guide the treatment of patients and optimise their ultimate outcome with the least amount of risk. I am confident that the diagnosis of exertional compartment syndrome is best done by performing pre and postexertional compartment pressure measures on all four compartments of both legs. Save the painful aspect of the testing, the risk related to additional needle pokes is negligible. The ultimate benefit is that on knowing which compartments are actually involved, the effected compartment can be released with little worry that the patient will have to return for additional surgery in the future due to an unrecognised, undiagnosed exertional compartment syndrome in an adjacent compartment.

\section{Competing interests None. \\ Provenance and peer review Commissioned; internally peer reviewed.}

\section{REFERENCES}

1. van den Brand J, Nelson T, Verleisdonk E. The diagnostic value of intracompartmental pressure measurement, magnetic resonance imaging and near-Infrared spectroscopy in chronic exertional compartment syndrome: a prospective study of 50 patients. Am J Sports Med 2005;33:699-704.

2. Tzortziou V, Maffulli N, Padhiar N. Diagnosis and management of chronic exertional compartment syndrome (CECS) in the United Kingdom. Clin J Sport Med 2006;16:209-13.

3. Pedowitz RA, Hargens AR, Mubarak SJ, et al. Modified criteria for the objective diagnosis of chronic compartment syndrome of the leg. Am J Sports Med 1990;18:35-40.

4. Gracia-Mata, Hidalgo-Ovejero A, Martinez-Grande M. Chronic exertional compartment syndrome of the legs in adolescents. J Pediatr Orthop 2001;21:328-34

5. Fraipont MJ, Adamson GJ. Chronic exertional compartment syndrome. J Am Acad Orthop Surg 2003;11:268-76. 